\title{
Skills upgrading for newly qualified surgeon: Is the district hospital in Kenya suitable?
}

Ojuka D., MBChB, MMed, General Surgeon, Kapenguria District Hospital, P.O. Box 63-30600, Kapenguria, Kenya, Email: dkinyuru@yahoo.com

\section{Abstract}

BACKGROUND: Surgical training in many settings involves acquisition of both knowledge and skill in an environment of adequate caseloads and dedicated supervision. With adequate surgical activity in these settings, the trainee's confidence is boosted to the point of independence. This skill acquisition is a continuous process, especially so for those who qualify from an exit training program like the one at the University of Nairobi (UON). The continuity is maintained for two years after the masters in surgery program under an experienced surgeon leading to registration by the medical board. This seems to have changed with the posting of newly qualified surgeons to the district hospitals, which has meant a transition from trainee to service provider in the absence of supervision.

OBJECTIVE: This study sought to document the caseload and type of surgical pathologies in the district hospital, the morbidities/mortality for cases operated on at the district, the proportion of the cases referred to higher level surgical centres and the reasons for referrals.

SETTING: The Kapenguria District Hospital $(\mathrm{KDH})$ in the Rift Valley province of Kenya.

METHODOLOGY: Records of all surgical patients attended to at the KDH between April 2007 and September 2007 were reviewed. Information collected included the nature of surgical pathology, investigations ordered and where these were done, surgical treatment offered, referrals, post-operative complications and lengths of stay. All operations were performed or supervised by the author. Data were entered onto an SPSS 12.0 software program and analyzed for summary data.

RESULTS: Two hundred and ninety patients were evaluated. The main surgical pathologies were general trauma, goitres and herniae. There were $29(10 \%)$ referrals to higher level facilities mainly due to lack of surgical instruments. Most investigations requested could not be done at $\mathrm{KDH}$. The morbidity and mortality rate for the group of surgical patients was $12.4 \%$ and $4.1 \%$ respectively. Hospital stay averaged 3.46 days.

CONCLUSION: The district hospital presents a potential setting for acquisition of surgical skills with its variety of surgical pathology and reasonable outcome data. However, the low volumes, lack of investigative capacity and surgical instruments compounded by absence of supervision, dictates that improvements in the system must be effected before the district hospital can be an ideal environment for the immediate post-residency training.

\section{Introduction}

Surgical training worldwide is based on apprenticeship for building of confidence, acquisition of knowledge and skills. This requires a balance between supervision by an experienced surgeon and operating in the absence of direct supervision in complex procedures. The training 
is at its most optimal where there is both the number and variety of surgical pathologies to go hand in hand with appropriate supervision (1). The weighted work load has been found to be more useful than case counts $(2,3)$ in working out assessment in surgical training. During the last three decades, individuals qualifying as surgeons from UON have benefitted as a result of supervision by experienced surgeons at provincial hospitals during the mandatory two years of supervised practice prior to registration. This model has changed since the government, as the principal sponsor of training, has decided to post the newly qualified surgeons to peripheral district hospitals where they find themselves as the sole surgeons. The question arises as to whether these district hospitals have the capacity in terms of caseloads and variety of surgical pathology as well as investigative capacity and availability of surgical instruments to be involved in the continuity of surgical training despite the lack of supervision.

\section{Materials and Methods}

Study Setting: Kapenguria district hospital is situated in northern part of Rift Valley provincein the West Pokot District in Kenya. The catchment area includes the Pokot North as well as Pokot Central District. West Pokot has a population of 310,000 . In total the catchment area population is about 500,000 people. The hospital is built along the Kitale-Lodwar highway. Surgical patients are admitted either as emergencies through the outpatient department/casualty or as elective cases through the surgical outpatient clinic. A few patients are managed as outpatients in the clinic and some as day care surgical cases. Due to the distances, terrain and transport problems, some patients who would otherwise be day cases are admitted for surgery.

During the study period, the author, who at the time had just been posted following completion of residency, operated or supervised the medical officers working under him in all surgical operations performed at $\mathrm{KDH}$.

Study design: This was a prospective observational study of six months between April 2007 and September 2007.
Patients and methods: The data were collected through a questionnaire. The data elements sought included: the final diagnosis, mode of admission, investigations required and whether these were performed at KDH, need for surgery, transfers to higher level facility and reasons, nature of surgical operation, post operative complications and length of stay.

The records were then entered into SPSS version 12.0 program for analysis. The results are summarized in terms of proportions and presented as tables and charts. Approval for the study was obtained from the Ministry of Health, division of standard and regulatory services.

\section{Results}

Two hundred and ninety patients were reviewed at the $\mathrm{KDH}$ surgical service between April and September 2007. This averaged about 48 patients per month. The most common surgical pathologies were general trauma 79 $(27.2 \%)$, goitre $34(11.7 \%)$ and herniae $15(5.2 \%)$ (Figure 1).

One hundred and twenty five $(43 \%)$ patients were admitted as emergency cases, 79 (27.25\%) as elective while 86 patients were reviewed and treated without admission.

Nineteen patients $(6.6 \%)$ were referred to a higher level facility from the point of first contact at $\mathrm{KDH}$. An additional ten $(3.4 \%)$ were transferred out after a period of treatment at $\mathrm{KDH}$. The reasons for the transfers included post thyroidectomy bleeding (one case) presumed to be due to a coagulopathy, breakdown of repair for perforated duodenal ulcer (one case) and long bone fractures (the rest of patients). Altogether, $90 \%$ of the patients received all their treatment at the $\mathrm{KDH}$.

About 15(52\%) of all the referred patients had sustained extremity fractures and transferred due to lack of orthopaedic instrument sets. Four infants were transferred because the available clinical officers lacked the competence to anaesthetize infants. Other reasons for referral included the need for further investigations, a subspecialist opinion and transfusion therapy with fresh frozen plasma for a patient with coagulopathy (Table 1). 


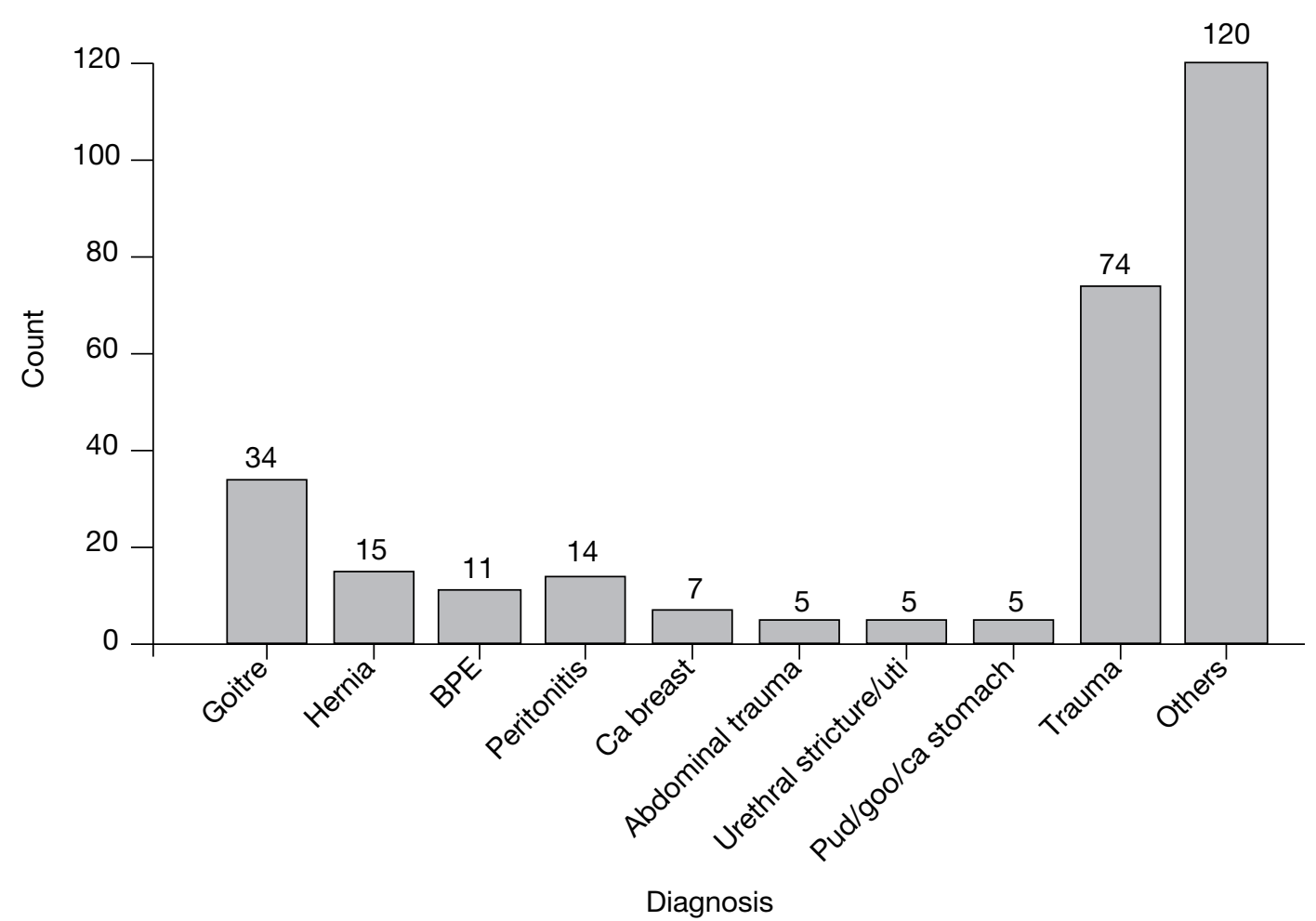

$(\mathrm{BPE}=$ benign prostate enlargement, $\mathrm{ca}=$ cancer, $\mathrm{pud}=$ peptic ulcer disease, goo $=$ gastric outlet obstruction, uti $=$ urinary tract infection)

Table 1: Reasons for referral

\begin{tabular}{lcc}
\hline Reason & Frequency & Percentage \\
\hline Lack of orthopaedic and trauma sets & 15 & 53.6 \\
Lack of anaesthetic expertise (for children) & 4 & 14.3 \\
Sub-specialty care (e.g in congenital malformations and brain tumours) & 6 & 21.4 \\
Lack of investigative tools & 2 & 7.1 \\
Lack of fresh blood & 1 & 3.6 \\
\hline Total & 28 & 100 \\
\hline
\end{tabular}

The investigative capacity of the hospital at this point was poor. Only the basic manual haemogram and urinalysis could be done at the hospital (Table 2).

Of the 290 patients, $174(60 \%)$ required surgery. Fifteen patients did not return to the clinic during the duration of the study. Of the remaining 159 patients, 140 had their surgeries performed at $\mathrm{KDH}$.

The major operations performed commonly were thyroidectomy 22 (15.1\%) and laparotomy $16(11.5 \%)$ (Table 3$)$. 
Table 2. Investigative capacity

\begin{tabular}{ll}
\hline Investigation & Place performed \\
\hline Plain radiography & Private (Kapenguria) \\
Prostatic specific antigen & Private (Eldoret) \\
Ultrasonography & Private(Kitale) \\
Endoscopy & Private (Eldoret) \\
Thyroid function test & Private (Eldoret) \\
Urea and electrolysis & Private (Eldoret and Kitale) \\
Blood sugar & KDH \\
Full haemogram & At the district Hospital (KDH) \\
Urinalysis & At the district Hospital (KDH) \\
Fine needle aspirate & Private (Eldoret) \\
Histology & Private (Eldoret) \\
\hline
\end{tabular}

Table 3: Surgical operations at KDH, April - Sept 2007.

\begin{tabular}{lcc}
\hline Type of surgery & Frequency & Percentage \\
\hline $\begin{array}{l}\text { Wound debridement } \\
\text { (including compound }\end{array}$ & 31 & 22.3 \\
fractures) & & \\
Surgical excision & 25 & 18.0 \\
Thyroidectomy & 22 & 15.1 \\
Laparotomy & 16 & 11.5 \\
Hernia repair & 11 & 7.9 \\
Prostatectomy & 7 & 3.6 \\
Mastectomy & 3 & 2.1 \\
Haemorrhoidectomy & 2 & 1.4 \\
Other procedures & 23 & 16.5 \\
\hline Total & 140 & 100 \\
\hline
\end{tabular}

Post operative complications occurred in 17 (12.1\%) patients. Themostfrequentcomplication was infection following laparotomy in two patients and compound fractures in the rest (Table 4).

Table 4: Post operative complications at KDH April Sept 2007

\begin{tabular}{lcc}
\hline Type of complication & Frequency & Percentage \\
\hline Infections & 9 & 52.9 \\
Burst abdomen & 3 & 17.6 \\
Post-operative bleeding & 3 & 17.6 \\
Post-operative & 1 & 5.9 \\
hoarseness & & \\
Atelectasis & 1 & 5.9 \\
\hline Total & 17 & 100 \\
\hline
\end{tabular}

The mortality rate was $4.1 \%$ (7 patients) - a 90 year old man who developed atelectasis post herniorrhaphy, 5 multiply-injured patients who developed multiple organ failures and one with prolonged intestinal obstruction. The average length of stay was 3.42 days (range of 1 to 46 days).

\section{Discussion}

The aim of any surgical training program is acquisition of knowledge, skills, adequate judgement and professionalism. In an exit training such as the one at the University of Nairobi, the skills and the confidence would be given more time to improve in an environment of moderated supervision where the new surgeon is given a leeway of making decisions for both elective and emergency surgical cases. Although the variety of surgical pathologies is appreciable at the $\mathrm{KDH}$, the volumes are not inspiring. Two hundred and ninety patients seen over six months translates to about ten patients per week and an even lower statistic on the week's theatre log. Given that the acquisition of confidence and skill is maximal where there is both the volume and case mix that go hand in hand with appropriate supervision (1), the ministry of health should re-examine the current strategy for the good of the trainees and patients. In other systems, it takes time before one is allowed to go out and practice as a consultant surgeon (4).

The capacity to train, apart from supervision, includes support services, availability of instruments and investigative facilities. The ability to investigate in a timely fashion allows early decisions for good surgical outcome. At the $\mathrm{KDH}$, timely surgical decision is not always possible as patients may seek the required investigations at facilities far away from the hospital. For a hospital situated along the highway, investing in trauma operative instruments would be necessary to reduce referrals and build confidence in trauma surgery.

In Australia and Ireland, the district hospital forms part and parcel of the rotations for surgical training $(3,4,5)$. Although it may be said that these are economically advanced 
countries and equipping hospitals not as big a challenge, it is possible to still equip our district hospitals with basic instruments so that, like in the countries mentioned, the district hospital can serve as the source of most surgical experience not only for the qualified surgeons but also for those still undergoing their surgical training. As shown in this study where morbidity and mortality rates of $12.2 \%$ and $4.1 \%$ were recorded, reasonable surgical outcomes can be achieved at the district hospital.

\section{References}

1. Charles C. Surgical training, supervision and service. Brit. Med. J. 1999; 318: 682-683.

2. Jones S.M. and Collins C.D. Caseload or work load? Scoring complexities of operative procedures as a means of analysing work load. Brit. Med. J. 1990; 301: 324-325.

3. Potter M.A., Griffiths J.M. Aitken R.J. and Crofts T.J. An objective assessment surgical training. Ann. R. Coll. Surg. Engl. 1996; 78 (Suppl): 11-13.

4. Bidawi A. Career focus: A career in Surgery. Brit. Med. J. 2001; 320: 217-260.

5. Kenneth W. and David B. Operative experience in the Victorian general surgical training programme. ANZ J. Surg. 2003; 73 (12): 1036-1040.

6. O' Leary D.P. and Collins C.D. Analysis of a year's general surgical activity in a District General Hospital. Ann. R. Coll.. Surg Engl. 1994; 76 (4 suppl): 176-81.

7. Davies M.G., Shine M.F, and Lennon F. Surgical emergencies in Ireland. An audit of the emergency surgical caseload of an Irish district general hospital. Ir. J. Med. Sci. 1991; 160(10): 303-306. 